\begin{tabular}{|c|l|}
\hline Title & Inverse scattering problem for time dependent Hartree Fock equations in the three body case \\
\hline Author(s) & Watanabe, Michiyuki \\
\hline Citation & $\begin{array}{l}\text { Journal of Mathematical Physics, 48(5), 053510 } \\
\text { https://doi.org/10.1063/1.2732171 }\end{array}$ \\
\hline Issue Date & $2007-05$ \\
\hline Doc URL & http://hdl.handle.net/2115/28008 \\
\hline Rights & Copyright $\odot 2007$ American Institute of Physics. \\
\hline Type & article \\
\hline File Information & JMP48.pdf \\
\hline
\end{tabular}

Instructions for use 


\title{
Inverse scattering problem for time dependent Hartree- Fock equations in the three-body case
}

\author{
Michiyuki Watanabe ${ }^{a)}$ \\ Department of Mathematics, Hokkaido University, Kita 10, Nishi 8, Kita-Ku, Sapporo, \\ Hokkaido 060-0810, Japan
}

(Received 22 September 2006; accepted 28 March 2007; published online 23 May 2007)

We consider an inverse scattering problem for time dependent Hartree-Fock equations in the three-body case. It will be shown that interaction potentials with simple forms are uniquely determined by the corresponding scattering data. We also give reconstruction formulas. () 2007 American Institute of Physics.

[DOI: $10.1063 / 1.2732171]$

\section{INTRODUCTION}

\section{A. Hartree-Fock equation}

Hartree equation and Hartree-Fock equation are nonlinear equations which are derived in order to obtain an approximate solution of an $N$-body linear Schrödinger equation. Let $v_{j k}(x), 1$ $\leqslant j<k \leqslant N$ be the interaction potentials between $j$ th and $k$ th particles. Then, the dynamics of the quantum mechanical system is given by the $N$-body Schrödinger equation

$$
i \partial_{t} \Phi_{N}=-\sum_{j=1}^{N} \Delta_{x_{j}} \Phi_{N}+\sum_{1 \leqslant j<k \leqslant N} v_{j k}\left(x_{j}-x_{k}\right) \Phi_{N}
$$

where $x_{j} \in \mathbf{R}^{n}$ is the position of the $j$ th particle and $\Delta_{x_{j}}$ is the $n$-dimensional Laplacian in coordinates $x_{j}$.

We brief the derivation of the Hartree equation and the Hartree-Fock equation. As for the details see, e.g., Dirac, ${ }^{2}$ Kramer and Saraceno. ${ }^{5}$ Assume that the wave function $\Phi_{N}$ is written by the separation of variables

$$
\Phi_{N}\left(t, x_{1}, \ldots, x_{N}\right)=u_{1}\left(t, x_{1}\right) u_{2}\left(t, x_{2}\right) \cdots u_{N}\left(t, x_{N}\right) .
$$

Substituting this wave function to the $N$-body Schrödinger equation [Eq. (1)] and using the variation principle, the following equation for $u_{j}(j=1, \ldots, N)$, which is called the time dependent Hartree equation, is derived:

$$
i \partial_{t} u_{j}=-\Delta u_{j}+\sum_{k \neq j}^{N}\left(v_{j k} *\left|u_{k}\right|^{2}\right) u_{j},
$$

where $*$ is the convolution. We note that the wave function [Eq. (2)] does not satisfy symmetry properties. If we consider a fermionic $N$-body system, the wave function must satisfy the antisymmetry property. Hartree-Fock equation is derived in order to improve the wave function [Eq. (2)] to satisfy the antisymmetry property. Assume that the wave function is written by the so-called Slater determinant,

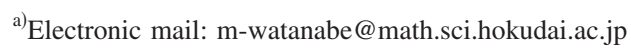




$$
\Phi_{N}\left(t, x_{1}, \ldots, x_{N}\right)=(N !)^{-1 / 2} \operatorname{det}\left(u_{j}\left(t, x_{k}\right)\right)_{1 \leqslant j, k \leqslant N} .
$$

Substituting this wave function to Eq. (1) and using variation principle, we obtain the following equation which is called the time dependent Hartree-Fock equation:

$$
i \partial_{t} u_{j}=-\Delta u_{j}+\sum_{k \neq j}^{N}\left(v_{j k} *\left|u_{k}\right|^{2}\right) u_{j}-\sum_{k \neq j}^{N}\left(v_{j k} * u_{j} \bar{u}_{k}\right) u_{k} .
$$

We note that solutions $u_{j}, j=1,2, \ldots, N$ of Eq. (5) must be linearly independent. If they are linearly dependent, then the wave function [Eq. (4)] vanishes and it has no physical meaning.

The aim of this paper is to show that interactions $v_{j k}(x)$ are uniquely determined by the scattering data, which is defined below, corresponding to the time dependent Hartree-Fock equation [Eq. (5)].

It should be mentioned that there are some results on inverse scattering problems for $N$-body Schrödinger equations. For instance, Wang ${ }^{15}$ showed that short-range interactions are uniquely determined by the free-channel to free-channel scattering matrix at high energies. It is proved by Enss and $\mathrm{Weder}^{3}$ that the high velocity limit of the scattering operator of an $N$-body system determines uniquely the potential. Novikov ${ }^{8}$ simplified methods of high energy inverse scattering for the $N$-body Schrödinger operator given by Wang and by Enss and Weder. Vasy ${ }^{13}$ showed that two-body pair potentials are reconstructed from the three-body scattering matrix when the perturbations are exponentially decreasing. Uhlmann and Vasy ${ }^{12}$ showed that if all unknown interactions are small in the appropriate sense, then the two-cluster to two-cluster scattering matrix given at low energies determines the Fourier transform of the effective interaction in a ball.

Our approach to inverse scattering problems for $N$-body systems is different from the abovementioned works. As far as the author is aware, there are no results available on inverse scattering problems for Hartree-Fock equations.

\section{B. Scattering for Equations (3) and (5)}

Assume that functions $v_{j k}(x)$ tend to zero sufficiently fast as $|x| \rightarrow \infty$. Then, we expect that there exist solutions $u_{j}$ of Eq. (3) or (5) which tend to free solutions $U_{j}^{( \pm)}(t, x)$ (solutions of the linear equation),

$$
i \partial_{t} U_{j}^{( \pm)}=-\Delta U_{j}^{( \pm)}, \quad U_{j}^{( \pm)}(0, x)=\phi_{j}^{( \pm)}(x),
$$

as $t \rightarrow \pm \infty$. In fact, if $v_{j k}(x)$ satisfies the condition

$$
\left|v_{j k}(x)\right| \leqslant c|x|^{-\sigma}, \quad 2 \leqslant \sigma \leqslant 4 \text { and } \sigma<n,
$$

then solutions of Eq. (3) or (5) tend to free solutions as $t \rightarrow \pm \infty$.

Theorem 1.1: There exists $\rho>0$ such that for any

$$
\phi_{j}^{(-)} \in \mathcal{D}_{\rho}:=\left\{\phi \in H^{1} ;\|\phi\|_{H^{1}}<\rho\right\}, \quad j=1,2, \ldots, N,
$$

there exists a unique solution of Eq. (3) or (5),

$$
u_{j} \in L^{3}\left(\mathbf{R} ; H^{1, q}\right) \cap L^{\infty}\left(\mathbf{R} ; H^{1}\right), \quad 1 / q=1 / 2+2 /(3 n),
$$

satisfying

$$
\lim _{t \rightarrow-\infty}\left\|u_{j}(t, \cdot)-U_{j}^{(-)}(t, \cdot)\right\|_{H^{1}}=0 .
$$

Moreover, for these solutions there exist $\phi_{j}^{(+)}:=U_{j}^{(+)}(x, 0)$ such that

$$
\lim _{t \rightarrow \infty}\left\|u_{j}(t, \cdot)-U_{j}^{(+)}(t, \cdot)\right\|_{H^{1}}=0 .
$$

The map $\mathbf{S}: \boldsymbol{\phi}^{(-)} \rightarrow \boldsymbol{\phi}^{(+)}$is called the scattering operator and defined by 


$$
S_{j}(\phi)(x)=\phi_{j}(x)+\frac{1}{i} \int_{\mathbf{R}} \mathrm{e}^{i t H_{0}} F_{j}(\boldsymbol{u}(t)) d t, \quad j=1, \ldots, N,
$$

where $\boldsymbol{\phi}^{( \pm)}={ }^{t}\left(\phi_{1}^{( \pm)}, \ldots, \phi_{N}^{( \pm)}\right), H_{0}=-\Delta, F_{j}(\boldsymbol{u}(t))$ denotes the nonlinearity of the equation, and $\boldsymbol{u}$ $={ }^{t}\left(u_{1}, \ldots, u_{N}\right)$ is a solution of equations. In the case of the Hartree equation [Eq. (3)] $F_{j}$ is given by

$$
F_{j}(\boldsymbol{u}(t))(x)=\sum_{k \neq j}^{N}\left(v_{j k} *\left|u_{k}\right|^{2}\right) u_{j},
$$

where $\boldsymbol{u}(t)$ is the solution of the Hartree equation [Eq. (3)]. In the case of the Hartree-Fock equation $F_{j}$ is given by

$$
F_{j}(\boldsymbol{u}(t))(x)=\sum_{k \neq j}^{N}\left(v_{j k} *\left|u_{k}\right|^{2}\right) u_{j}-\sum_{k \neq j}^{N}\left(v_{j k} * u_{j} \bar{u}_{k}\right) u_{k},
$$

where $\boldsymbol{u}(t)$ is the solution of the Hartree-Fock equation [Eq. (5)]. We call the set of functions $\left\{S_{j}(\boldsymbol{\phi})(x), \phi_{j}(x)\right\}(j=1, \ldots, N)$ the scattering data. Theorem 1.1 is essentially due to Mochizuki. ${ }^{6} \mathrm{~A}$ similar result was obtained by Wada. ${ }^{14}$

\section{Problem and result}

The problem which we consider in this paper is: Given functions $S_{j}(\boldsymbol{\phi})(x)$ and $\phi_{j}(x)$, find unknown functions $v_{j k}(x), 1 \leqslant j<k \leqslant N$. Mathematically speaking, solve the integral equation [Eq. (7)] for $v_{j k}(x)$, given function $S_{j}(\boldsymbol{\phi})(x)$ and $\phi_{j}(x)$.

It seems to be difficult to solve this problem in the general situation. One of the reasons is that the problem is to determine unknown functions $v_{j k}$ from given functions $\left\{S_{j}(\boldsymbol{\phi})(x), \phi_{j}(x)\right\}, 1 \leqslant j$ $<k \leqslant N$; namely, the number of unknown functions $\left({ }_{N} C_{2}\right)$ is more than the number of given functions $(=N)$ in general. Only for the three-body case are they equal. Moreover, the integral equation [Eq. (7)] is nonlinear for $v_{j k}(x)$. Hence, it is very difficult technically to solve Eq. (7) explicitly. Therefore, as a first attack, we consider a simple case, namely, the three-body case $N$ $=3$ for simple pair interaction potentials,

$$
v_{j k}(x)=v_{k j}(x)=\lambda_{j k}|x|^{-\sigma_{j k},} \quad 1 \leqslant j, k \leqslant 3,
$$

where $\lambda_{j k} \in \mathbf{R}$ and $\sigma_{j k}$ are constants such that

$$
2 \leqslant \sigma_{j k} \leqslant 4 \text { and } \sigma_{j k}<n .
$$

In this situation, we can show that the scattering data uniquely determine interactions, that is, $\lambda_{j k}$ and $\sigma_{j k}, 1 \leqslant j, k \leqslant 3$. In other words, we can determine the size and decay rate of interactions from the scattering data.

To state our results we introduce some notations. Put

$$
\mathcal{T}_{j}[\boldsymbol{\phi}]:=\lim _{\varepsilon \rightarrow 0} \frac{i}{\varepsilon^{3}}\left(\left[S_{j}-I_{j}\right](\boldsymbol{\phi}), \phi_{j}\right)_{L^{2}}, \quad j=1,2,3,
$$

where $\boldsymbol{\phi}=\left(\phi_{1}, \phi_{2}, \phi_{3}\right), I_{j}(\boldsymbol{\phi})=\phi_{j}$, and $(\cdot, \cdot)_{L^{2}}$ denotes the inner product in $L^{2}\left(\mathbf{R}^{n}\right)$. We denote by $\Upsilon$ the set of functions $f(x)$ in the Schwartz space whose Fourier transform $\hat{f} \in C_{0}^{\infty}\left(\mathbf{R}^{n}\right)$. In the case where $N=3, \lambda_{j k}$ and $\sigma_{j k}$ are simply denoted $\lambda_{j}$ and $\sigma_{j}$, respectively. In this paper we show the following.

Theorem 1.2: There exist

$$
\boldsymbol{\phi} \in \mathcal{B}:=\left\{\boldsymbol{\phi}=\left(\phi_{1}, \phi_{2}, \phi_{3}\right), \phi_{j} \in \Upsilon ; \phi_{j} \neq c \phi_{k}, 1 \leqslant j, k \leqslant 3, c \in \mathbf{R}\right\}
$$

such that following formulas hold for any positive $R \neq 1$ : 


$$
\begin{gathered}
\sigma_{1}=2 n+2+\log _{R}\left(\frac{\mathcal{T}_{1}\left[\boldsymbol{\phi}_{R}\right]+\mathcal{T}_{2}\left[\boldsymbol{\phi}_{R}\right]-\mathcal{T}_{3}\left[\boldsymbol{\phi}_{R}\right]}{\mathcal{T}_{1}[\boldsymbol{\phi}]+\mathcal{T}_{2}[\boldsymbol{\phi}]-\mathcal{T}_{3}[\boldsymbol{\phi}]}\right), \\
\sigma_{2}=2 n+2+\log _{R}\left(\frac{\mathcal{T}_{2}\left[\boldsymbol{\phi}_{R}\right]+\mathcal{T}_{3}\left[\boldsymbol{\phi}_{R}\right]-\mathcal{T}_{1}\left[\boldsymbol{\phi}_{R}\right]}{\mathcal{T}_{2}[\boldsymbol{\phi}]+\mathcal{T}_{3}[\boldsymbol{\phi}]-\mathcal{T}_{1}[\boldsymbol{\phi}]}\right), \\
\sigma_{3}=2 n+2+\log _{R}\left(\frac{\mathcal{T}_{3}\left[\boldsymbol{\phi}_{R}\right]+\mathcal{T}_{1}\left[\boldsymbol{\phi}_{R}\right]-\mathcal{T}_{2}\left[\boldsymbol{\phi}_{R}\right]}{\mathcal{T}_{3}[\boldsymbol{\phi}]+\mathcal{T}_{1}[\boldsymbol{\phi}]-\mathcal{T}_{2}[\boldsymbol{\phi}]}\right),
\end{gathered}
$$

where $\boldsymbol{\phi}_{R}(x)=\boldsymbol{\phi}(R x)$, and

$$
\begin{aligned}
& \lambda_{1}=\frac{1}{2\left(a_{1}-b_{1}\right)}\left(\mathcal{T}_{1}[\boldsymbol{\phi}]+\mathcal{T}_{2}[\boldsymbol{\phi}]-\mathcal{T}_{3}[\boldsymbol{\phi}]\right), \\
& \lambda_{2}=\frac{1}{2\left(a_{2}-b_{2}\right)}\left(\mathcal{T}_{2}[\boldsymbol{\phi}]+\mathcal{T}_{3}[\boldsymbol{\phi}]-\mathcal{T}_{1}[\boldsymbol{\phi}]\right), \\
& \lambda_{3}=\frac{1}{2\left(a_{3}-b_{3}\right)}\left(\mathcal{T}_{3}[\boldsymbol{\phi}]+\mathcal{T}_{1}[\boldsymbol{\phi}]-\mathcal{T}_{2}[\boldsymbol{\phi}]\right),
\end{aligned}
$$

where

$$
\begin{aligned}
& a_{1}=\int_{\mathbf{R}} \int_{\mathbf{R}^{n}}\left(|x|^{-\sigma_{1}} *\left|U_{1}(t, \cdot)\right|^{2}\right)(x)\left|U_{2}(t, x)\right|^{2} d x d t, \\
& a_{2}=\int_{\mathbf{R}} \int_{\mathbf{R}^{n}}\left(|x|^{-\sigma_{2}} *\left|U_{2}(t, \cdot)\right|^{2}\right)(x)\left|U_{3}(t, x)\right|^{2} d x d t, \\
& a_{3}=\int_{\mathbf{R}} \int_{\mathbf{R}^{n}}\left(|x|^{-\sigma_{3}} *\left|U_{3}(t, \cdot)\right|^{2}\right)(x)\left|U_{1}(t, x)\right|^{2} d x d t, \\
& b_{1}=\int_{\mathbf{R}} \int_{\mathbf{R}^{n}}\left(|x|^{-\sigma_{1}} * U_{1}(t, \cdot) \overline{U_{2}}(t, \cdot)\right)(x) \overline{U_{1}}(t, x) U_{2}(t, x) d x d t, \\
& b_{2}=\int_{\mathbf{R}} \int_{\mathbf{R}^{n}}\left(|x|^{-\sigma_{2}} * U_{2}(t, \cdot) \overline{U_{3}}(t, \cdot)\right)(x) \overline{U_{2}}(t, x) U_{3}(t, x) d x d t, \\
& b_{3}=\int_{\mathbf{R}} \int_{\mathbf{R}^{n}}\left(|x|^{-\sigma_{3}} * U_{1}(t, \cdot) \overline{U_{3}}(t, \cdot)\right)(x) \overline{U_{1}}(t, x) U_{3}(t, x) d x d t .
\end{aligned}
$$

Remark 1: As has been mentioned, the solution of Eq. (5) must be linearly independent. Hence, the condition $\phi_{j} \neq c \phi_{k}, 1 \leqslant j, k \leqslant 3$ in Theorem 1.2 seems to be natural in the physical point of view. On the other hand, linearly independence of the solution for the Hartree equation [Eq. (3)] is not necessary and the formulas for the Hartree equation, in fact, hold for any $\phi_{j} \in H^{1}, j$ $=1,2,3$. The result for the Hartree equation case is given in Sec. IV. 


\section{Known results}

The Hartree equation and the Hartree-Fock equation are nonlinear equations. Hence, our problem is an inverse scattering problem for nonlinear equations. In 1973, Morawetz and Strauss ${ }^{7}$ considered the inverse scattering problem for the nonlinear Klein-Gordon equation

$$
u_{t t}-\Delta u+m^{2} u+g u^{3}=0, \quad(t, x) \in \mathbf{R} \times \mathbf{R}^{3},
$$

where $m>0, g>0$. It was proved that the coupling constant $g$ is uniquely determined by the corresponding scattering operator. Moreover, a reconstruction formula was given. They also proved that this result is extended to the equation where $g u^{3}$ is replaced by any interaction function $f(u)$ which is odd and analytic. Since $f(u)$ is analytic, it is enough to determine each coefficient in the expansion of $f(z)$. These results, therefore, mean that a scattering operator uniquely determines constant coefficients of nonlinear equations.

Strauss ${ }^{11}$ extended the above results to nonlinear Schrödinger equations and nonlinear KleinGordon equations with a variable coefficient. He proved that for the following nonlinear Schrödinger equation with power-type nonlinearity,

$$
i \partial_{t} u=-\Delta u+V(x)|u|^{p-1} u, \quad(t, x) \in \mathbf{R} \times \mathbf{R}^{n},
$$

the coefficient $V(x)$ of the nonlinear part is uniquely determined by the corresponding scattering operator and gave a reconstruction formula under the suitable condition on $p$ and $V(x)$. This result was also applied to the nonlinear Klein-Gordon equation with the power-type nonlinearity.

Weder ${ }^{19-24}$ has extended the above results to more general nonlinear Schrödinger and nonlinear Klein-Gordon equations with power-type nonlinearity. He proved that the corresponding scattering operator uniquely determines not only the variable coefficient of nonlinear part, but also the one of linear part. Moreover, reconstruction formulas were given. In Weder ${ }^{25,26}$ the scattering problem and the inverse scattering problem for the nonlinear Schrödinger equation with powertype nonlinearity were considered on the half line.

The application of the above results to a Hartree-type equation, whose nonlinearity is the form $\left(v *|u|^{2}\right) u$, was studied by Watanabe. ${ }^{16-18}$ Contrary to the power-type case, a reconstruction formula for Hartree-type nonlinearities, i.e., $v$, has not been given yet. In a particular case $v$ $=\lambda|x|^{-\sigma}$, where $\lambda$ and $\sigma$ are constants, reconstruction formulas were given by Watanabe. ${ }^{18}$ In the case of general function $v(x)$, a uniqueness result was proven-by Sasaki and Watanabe ${ }^{10}$ for the Hartree equation [Eq. (3)] and the nonlinear Klein-Gordon equation. Sasaki ${ }^{9}$ extended the results in Watanabe ${ }^{18}$ to the equation where a constant $\lambda$ is replaced by a function $\lambda(x, y)$.

We should mention that inverse scattering problems for stationary nonlinear Schrödinger equations (nonlinear Helmholtz equations) with power-type nonlinearity were considered by Jalade $^{4}$ and by Aktosun et al. ${ }^{1}$

Theorem 1.2 is an application of the result in Watanabe ${ }^{18}$ to the Hartree-Fock equation [Eq. (5)]. The difficulty of the inverse scattering problem for the Hartree-Fock equation is to characterize $\phi_{j}, j=1,2,3$ such that formulas in Theorem 1.2 hold. By controlling supports of function $\phi_{j}(x)$, an existence of the scattering data satisfying formulas in Theorem 1.2 is proved.

\section{PRELIMINARY LEMMAS}

We need some notations and some lemmas. Denote by

$$
\boldsymbol{U}(t)=\left(U_{1}(t, x), U_{2}(t, x), U_{3}(t, x)\right)
$$

the free solution with the initial data $\boldsymbol{\phi}(x)=\left(\phi_{1}(x), \phi_{2}(x), \phi_{3}(x)\right)$. We will write $U_{j}(t, x)$ $=\left(\mathrm{e}^{-i t H_{0}} \phi_{j}\right)(x)$.

Lemma 2.1: Let $v_{j k}(x)$ satisfy Eq. (6). Then, for any $\phi_{j} \in H^{1}, j=1,2,3$, the small amplitude limit of the scattering operator $\mathcal{T}_{j}[\boldsymbol{\phi}]$ exists and 


$$
\mathcal{T}_{j}[\boldsymbol{\phi}]=\int_{\mathbf{R}} \int_{\mathbf{R}^{n}} \overline{U_{j}(t, x)} F_{j}(\boldsymbol{U}(t)) d x d t,
$$

where $F_{j}(\boldsymbol{U}(t))$ is Eq. (8) or (9).

Proof: The Hartree nonlinearity case [Eq. (8)] proved in Sasaki and Watanabe. ${ }^{10}$ Noting that

$$
\left[v_{j k} * u_{j} \bar{u}_{k}\right] u_{k}=\left[v_{j k} * u_{j} \bar{u}_{k}\right] w_{k}+\left[v_{j k} * \bar{u}_{k} w_{j}\right] U_{k}+\left[v_{j k} * \bar{w}_{k} U_{j}\right] U_{k}+\left[v_{j k} * U_{j} \bar{U}_{k}\right] U_{k},
$$

where $w_{j}=u_{j}-U_{j}$, we can prove identity (13) similarly for case (8).

Lemma 2.2: Let $2 \leqslant \sigma \leqslant 4$ and $\sigma<n$. Then, for any $\phi_{j}, \phi_{k} \in H^{1}(j, k \in \mathbf{N})$

$$
B=\int_{\mathbf{R}} \int_{\mathbf{R}^{n}}\left(|x|^{-\sigma} * U_{j}(t, \cdot) \overline{U_{k}}(t, \cdot)\right)(x) \overline{U_{j}}(t, x) U_{k}(t, x) d x d t
$$

is real number.

Proof: The case $j=k$ is obvious. If $j \neq k$, then Fubini's theorem allows us to show that

$$
\begin{aligned}
B & =\int_{\mathbf{R}} \int_{\mathbf{R}_{x}^{n}} \int_{\mathbf{R}_{y}^{n}}|x-y|^{-\sigma} U_{j}(t, y) \overline{U_{k}}(t, y) \overline{U_{j}}(t, x) U_{k}(t, x) d y d x d t \\
& =\int_{\mathbf{R}} \int_{\mathbf{R}_{y}^{n}}\left(|y|^{-\sigma} * \overline{U_{j}}(t, \cdot) U_{k}(t, \cdot)\right)(y) U_{j}(t, y) U_{k}(t, y) d y d t=\bar{B} .
\end{aligned}
$$

Lemma 2.3: For any $R>0$ and $\phi \in H^{1}$ we have

$$
\left(e^{-i t H_{0}} \phi_{R}\right)(x)=\left(\mathrm{e}^{-i R^{2} t H_{0}} \phi\right)(R x)
$$

and

$$
\int_{\mathbf{R}^{n}}\left(|x|^{-\sigma} * \phi_{R}\right) \phi_{R}(x) d x=R^{\sigma-2 n} \int_{\mathbf{R}^{n}}\left(|x|^{-\sigma} * \phi\right) \phi(x) d x .
$$

Proof: These identities follow from a straightforward computation. See, e.g., Watanabe. ${ }^{18}$

\section{PROOF OF THEOREM 1.2} write

We denote by $\mathcal{F}$ the Fourier transform. Put $V_{j}(x)=\lambda_{j}|x|^{-\sigma_{j}}$. In the case where $N=3$ we can

$$
\left\{\begin{array}{l}
F_{1}(\boldsymbol{u})(x)=\left\{\left(V_{1} *\left|u_{2}\right|^{2}\right)+\left(V_{3} *\left|u_{3}\right|^{2}\right)\right\} u_{1}-\left\{\left(V_{1} * \bar{u}_{2} u_{1}\right) u_{2}+\left(V_{3} * \bar{u}_{3} u_{1}\right) u_{3}\right\}, \\
F_{2}(\boldsymbol{u})(x)=\left\{\left(V_{1} *\left|u_{1}\right|^{2}\right)+\left(V_{2} *\left|u_{3}\right|^{2}\right)\right\} u_{2}-\left\{\left(V_{1} * \bar{u}_{1} u_{2}\right) u_{1}+\left(V_{2} * \bar{u}_{3} u_{2}\right) u_{3}\right\}, \\
F_{3}(\boldsymbol{u})(x)=\left\{\left(V_{3} *\left|u_{1}\right|^{2}\right)+\left(V_{2} *\left|u_{2}\right|^{2}\right)\right\} u_{3}-\left\{\left(V_{3} * \bar{u}_{1} u_{3}\right) u_{1}+\left(V_{2} * \bar{u}_{2} u_{3}\right) u_{2}\right\} .
\end{array}\right.
$$

It follows from Lemma 2.1 and Lemma 2.2 that

$$
\left\{\begin{array}{l}
\mathcal{T}_{1}[\boldsymbol{\phi}]=\lambda_{1} a_{1}+\lambda_{3} a_{3}-\lambda_{1} b_{1}-\lambda_{3} b_{3} \\
\mathcal{T}_{2}[\boldsymbol{\phi}]=\lambda_{1} a_{1}+\lambda_{2} a_{2}-\lambda_{1} b_{1}-\lambda_{2} b_{2} \\
\mathcal{T}_{3}[\boldsymbol{\phi}]=\lambda_{3} a_{3}+\lambda_{2} a_{2}-\lambda_{3} b_{3}-\lambda_{2} b_{2}
\end{array}\right.
$$

A simple computation shows that 


$$
\left\{\begin{array}{l}
\mathcal{T}_{1}[\boldsymbol{\phi}]+\mathcal{T}_{2}[\boldsymbol{\phi}]-\mathcal{T}_{3}[\boldsymbol{\phi}]=2 \lambda_{1}\left(a_{1}-b_{1}\right) \\
\mathcal{T}_{2}[\boldsymbol{\phi}]+\mathcal{T}_{3}[\boldsymbol{\phi}]-\mathcal{T}_{1}[\boldsymbol{\phi}]=2 \lambda_{2}\left(a_{2}-b_{2}\right) \\
\mathcal{T}_{3}[\boldsymbol{\phi}]+\mathcal{T}_{1}[\boldsymbol{\phi}]-\mathcal{T}_{2}[\boldsymbol{\phi}]=2 \lambda_{3}\left(a_{3}-b_{3}\right)
\end{array}\right.
$$

We then have formulas for $\lambda_{j}$ if $a_{j}-b_{j} \neq 0, j=1,2,3$.

We would like to show by reduction to absurdity that there exist $\boldsymbol{\phi} \in \mathcal{B}$ such that $a_{1} \neq b_{1}$. The proof is similar for $a_{2} \neq b_{2}$ and $a_{3} \neq b_{3}$. Assume that $a_{1}=b_{1}$ for any $\boldsymbol{\phi} \in \mathcal{B}$. Then, it follows from Plancherel's theorem that the identity

$$
\int_{\mathbf{R}} \int_{\mathbf{R}^{n}} \hat{V}_{1}(\xi)\left\{\mathcal{F}\left(\left|U_{2}\right|^{2}\right) \overline{\mathcal{F}\left(\left|U_{1}\right|^{2}\right)}-\left|\mathcal{F}\left(\bar{U}_{2} U_{1}\right)\right|^{2}\right\} \mathrm{d} \xi \mathrm{d} t=0
$$

holds for any $\boldsymbol{\phi} \in \mathcal{B}$, where $\hat{V}_{1}(\xi)=C|\xi|^{-n+\sigma}$. We note that if $\phi_{1}(x) \neq c \phi_{2}(x)$ for any $c \in \mathbf{R}$ and $x$ $\in \mathbf{R}^{n}$, then

$$
\mathcal{F}\left(\left|U_{2}\right|^{2}\right) \overline{\mathcal{F}\left(\left|U_{1}\right|^{2}\right)} \equiv\left|\mathcal{F}\left(\overline{U_{2}} U_{1}\right)\right|^{2} .
$$

In fact, assume that they are equal. Then, from the identity $\mathcal{F}\left(\left|U_{j}\right|^{2}\right)(0,0)=\left\|\phi_{j}\right\|_{L^{2}}^{2}$ we have $\left\|\phi_{1}\right\|_{L^{2}}\left\|\phi_{2}\right\|_{L^{2}}=\left|\left(\phi_{2}, \phi_{1}\right)_{L^{2}}\right|$ at the point $(t, \xi)=(0,0)$. This contradicts the linearly independence of $\phi_{1}$ and $\phi_{2}$.

We denote by $B_{a}(b)$ a ball with center $b$ and radius $a$. Taking $\boldsymbol{\psi} \in \mathcal{B}$ such that $\operatorname{supp} \hat{\psi}_{j}(\xi) \subset B_{\varepsilon_{j}}\left(\chi_{j}\right), j=1,2$, we find

$$
\operatorname{supp}_{t, \xi} \mathcal{F}\left(\bar{U}_{2} U_{1}\right)(t, \xi) \subset \mathbf{R} \times B_{\varepsilon_{1}+\varepsilon_{2}}\left(\chi_{1}-\chi_{2}\right) .
$$

We write $\varepsilon=\min \left\{\varepsilon_{1}, \varepsilon_{2}\right\}$. Since $\operatorname{supp} \mathcal{F}\left(\left|U_{j}\right|^{2}\right)(t, \xi) \subset \mathbf{R} \times B_{2 \varepsilon_{j}}(0)$,

$$
\operatorname{supp}_{t, \xi} \mathcal{F}\left(\left|U_{2}\right|^{2}\right)(t, \xi) \overline{\mathcal{F}\left(\left|U_{1}\right|^{2}\right)(t, \xi)} \subset \mathbf{R} \times B_{2 \varepsilon}(0) .
$$

Note that $\left|\mathcal{F}\left(\bar{U}_{j} U_{k}\right)(t, \xi)\right| \leqslant\left\|\psi_{j}\right\|_{L^{2}}\left\|\psi_{k}\right\|_{L^{2}}$. Thus, we can choose $\varepsilon_{1}, \varepsilon_{2}, \chi_{1}$, and $\chi_{2}$, so that

$$
\left|\int_{\mathbf{R}} \int_{\mathbf{R}^{n}} \hat{V}_{1}(\xi) \mathcal{F}\left(\left|U_{2}\right|^{2}\right) \overline{\mathcal{F}\left(\left|U_{1}\right|^{2}\right)} \mathrm{d} \xi \mathrm{d} t\right|<\int_{\mathbf{R}} \int_{\mathbf{R}^{n}} \hat{V}_{1}(\xi)\left|\mathcal{F}\left(\overline{U_{2}} U_{1}\right)\right|^{2} \mathrm{~d} \xi \mathrm{d} t .
$$

This contradicts Eq. (16).

We would like to derive formulas for $\sigma_{j}$. By virtue of Lemma 2.3 and Eq. (15), it follows from a straightforward computation that for any $R>0$ and $\phi \in H^{1}$ we have

$$
\left\{\begin{array}{l}
\mathcal{T}_{1}\left[\boldsymbol{\phi}_{R}\right]+\mathcal{T}_{2}\left[\boldsymbol{\phi}_{R}\right]-\mathcal{T}_{3}\left[\boldsymbol{\phi}_{R}\right]=R^{\sigma_{1}-2 n-2}\left(\mathcal{T}_{1}[\boldsymbol{\phi}]+\mathcal{T}_{2}[\boldsymbol{\phi}]-\mathcal{T}_{3}[\boldsymbol{\phi}]\right), \\
\mathcal{T}_{2}\left[\boldsymbol{\phi}_{R}\right]+\mathcal{T}_{3}\left[\boldsymbol{\phi}_{R}\right]-\mathcal{T}_{1}\left[\boldsymbol{\phi}_{R}\right]=R^{\sigma_{2}-2 n-2}\left(\mathcal{T}_{2}[\boldsymbol{\phi}]+\mathcal{T}_{3}[\boldsymbol{\phi}]-\mathcal{T}_{1}[\boldsymbol{\phi}]\right), \\
\mathcal{T}_{3}\left[\boldsymbol{\phi}_{R}\right]+\mathcal{T}_{1}\left[\boldsymbol{\phi}_{R}\right]-\mathcal{T}_{2}\left[\boldsymbol{\phi}_{R}\right]=R^{\sigma_{3}-2 n-2}\left(\mathcal{T}_{3}[\boldsymbol{\phi}]+\mathcal{T}_{1}[\boldsymbol{\phi}]-\mathcal{T}_{2}[\boldsymbol{\phi}]\right) .
\end{array}\right.
$$

As has been proved, there exists a $\boldsymbol{\phi} \in \mathcal{B}$ such that

$$
\mathcal{T}_{j}[\boldsymbol{\phi}]+\mathcal{T}_{k}[\boldsymbol{\phi}]-\mathcal{T}_{l}[\boldsymbol{\phi}]=2 \lambda_{j}\left(a_{j}-b_{j}\right) \neq 0, \quad 1 \leqslant j \neq k \neq l \leqslant 3 .
$$

Thus, formulas for $\sigma_{j}, j=1,2,3$ are obtained.

The proof is complete.

\section{REMARK}

In the case of Hartree equation [Eq. (3)], we do not need to assume the linear independence of each component of $\boldsymbol{\phi}$. In fact, for case (8), we have 


$$
\mathcal{T}_{1}[\boldsymbol{\phi}]=\lambda_{1} a_{1}+\lambda_{3} a_{3}, \quad \mathcal{T}_{2}[\boldsymbol{\phi}]=\lambda_{1} a_{1}+\lambda_{2} a_{2}, \quad \mathcal{T}_{3}[\boldsymbol{\phi}]=\lambda_{2} a_{2}+\lambda_{3} a_{3} .
$$

Then, a simple computation shows that

$$
\begin{gathered}
\mathcal{T}_{1}[\boldsymbol{\phi}]+\mathcal{T}_{2}[\boldsymbol{\phi}]-\mathcal{T}_{3}[\boldsymbol{\phi}]=2 \lambda_{1} a_{1}, \quad \mathcal{T}_{2}[\boldsymbol{\phi}]+\mathcal{T}_{3}[\boldsymbol{\phi}]-\mathcal{T}_{1}[\boldsymbol{\phi}]=2 \lambda_{2} a_{2}, \\
\mathcal{T}_{3}[\boldsymbol{\phi}]+\mathcal{T}_{1}[\boldsymbol{\phi}]-\mathcal{T}_{2}[\boldsymbol{\phi}]=2 \lambda_{3} a_{3} .
\end{gathered}
$$

If $\phi_{j} \neq 0$, then obviously $a_{j} \neq 0,1 \leqslant j \leqslant 3$. Hence, we obtain the following result by the same computation as we did to derive the formulas in Theorem 1.2.

Theorem 4.1: For any $0 \neq \phi_{j} \in H^{1}, 1 \leqslant j \leqslant 3$ and any $1 \neq R>0$, we have

$$
\begin{gathered}
\sigma_{1}=2 n+2+\log _{R}\left(\frac{\mathcal{T}_{1}\left[\boldsymbol{\phi}_{R}\right]+\mathcal{T}_{2}\left[\boldsymbol{\phi}_{R}\right]-\mathcal{T}_{3}\left[\boldsymbol{\phi}_{R}\right]}{\mathcal{T}_{1}[\boldsymbol{\phi}]+\mathcal{T}_{2}[\boldsymbol{\phi}]-\mathcal{T}_{3}[\boldsymbol{\phi}]}\right), \\
\sigma_{2}=2 n+2+\log _{R}\left(\frac{\mathcal{T}_{2}\left[\boldsymbol{\phi}_{R}\right]+\mathcal{T}_{3}\left[\boldsymbol{\phi}_{R}\right]-\mathcal{T}_{1}\left[\boldsymbol{\phi}_{R}\right]}{\mathcal{T}_{2}[\boldsymbol{\phi}]+\mathcal{T}_{3}[\boldsymbol{\phi}]-\mathcal{T}_{1}[\boldsymbol{\phi}]}\right), \\
\sigma_{3}=2 n+2+\log _{R}\left(\frac{\mathcal{T}_{3}\left[\boldsymbol{\phi}_{R}\right]+\mathcal{T}_{1}\left[\boldsymbol{\phi}_{R}\right]-\mathcal{T}_{2}\left[\boldsymbol{\phi}_{R}\right]}{\mathcal{T}_{3}[\boldsymbol{\phi}]+\mathcal{T}_{1}[\boldsymbol{\phi}]-\mathcal{T}_{2}[\boldsymbol{\phi}]}\right),
\end{gathered}
$$

where $\boldsymbol{\phi}_{R}(x)=\boldsymbol{\phi}(R x)$, and

$$
\begin{aligned}
& \lambda_{1}=\frac{1}{2 a_{1}}\left(\mathcal{T}_{1}[\boldsymbol{\phi}]+\mathcal{T}_{2}[\boldsymbol{\phi}]-\mathcal{T}_{3}[\boldsymbol{\phi}]\right), \\
& \lambda_{2}=\frac{1}{2 a_{2}}\left(\mathcal{T}_{2}[\boldsymbol{\phi}]+\mathcal{T}_{3}[\boldsymbol{\phi}]-\mathcal{T}_{1}[\boldsymbol{\phi}]\right), \\
& \lambda_{3}=\frac{1}{2 a_{3}}\left(\mathcal{T}_{3}[\boldsymbol{\phi}]+\mathcal{T}_{1}[\boldsymbol{\phi}]-\mathcal{T}_{2}[\boldsymbol{\phi}]\right),
\end{aligned}
$$

where

$$
\begin{aligned}
& a_{1}=\int_{\mathbf{R}} \int_{\mathbf{R}^{n}}\left(|x|^{-\sigma_{1} *}\left|U_{1}(t, \cdot)\right|^{2}\right)(x)\left|U_{2}(t, x)\right|^{2} d x d t, \\
& a_{2}=\int_{\mathbf{R}} \int_{\mathbf{R}^{n}}\left(|x|^{-\sigma_{2}} *\left|U_{2}(t, \cdot)\right|^{2}\right)(x)\left|U_{3}(t, x)\right|^{2} d x d t, \\
& a_{3}=\int_{\mathbf{R}} \int_{\mathbf{R}^{n}}\left(|x|^{-\sigma_{3}} *\left|U_{3}(t, \cdot)\right|^{2}\right)(x)\left|U_{1}(t, x)\right|^{2} d x d t .
\end{aligned}
$$

\footnotetext{
${ }^{1}$ Aktosun, T., Papanicolaou, V. G., and Zisis, V., "Inverse scattering on the line for a generalized nonlinear Schrödinger equation," Inverse Probl. 20, 1267-1280 (2004).

${ }^{2}$ Dirac, P. A. M., The Principles of Quantum Mechanics, 4th ed. (Oxford University Press, Oxford, 1958).

${ }^{3}$ Enss, V., and Weder, R., "The geometrical approach to multidimensional inverse scattering," J. Math. Phys. 36, 39023921 (1995).

${ }^{4}$ Jalade, E., "Inverse problem for a nonlinear Helmholtz equation," Ann. Inst. Henri Poincare, Anal. Non Lineaire 21, 517-531 (2004).

${ }^{5}$ Kramer, P., and Saraceno, M., "Geometry of the time-dependent variational principle in quantum mechanics," Lect.
} 
Notes Phys. 140, 71-76 (1981).

${ }^{6}$ Mochizuki, K., "On small data scattering with cubic convolution nonlinearity," J. Math. Soc. Jpn. 41, 143-160 (1989).

${ }^{7}$ Morawetz, C. S. and Strauss, W. A., "On a nonlinear scattering operator," Commun. Pure Appl. Math. 26, 47-54 (1973).

${ }^{8}$ Novikov, R. G., "On inverse scattering for the $N$-body Schrödinger equation," J. Funct. Anal. 159, $492-536$ (1998).

${ }^{9}$ Sasaki, H., "The inverse scattering problem for Schrödinger and Klein-Gordon equations with a nonlocal nonlinearity," Nonlinear Anal. Theory, Methods Appl. 66, 1770-1781 (2007).

${ }^{10}$ Sasaki, H., and Watanabe, M., "Uniqueness on identification of cubic convolution nonlinearity," J. Math. Anal. Appl. 309, 294-306 (2005)

${ }^{11}$ Strauss, W. A., "Non linear scattering theory," in Scattering Theory in Mathematical Physics, edited by Lavita, J. A., and Marchand, J.-P. (Reidel, Dordrecht, 1974), pp. 53-78.

${ }^{12}$ Uhlmann, G., and Vasy, A., "Low-energy inverse problems in three-body scattering," Inverse Probl. 18, 719-736 (2002).

${ }^{13}$ Vasy, A., "Structure of the resolvent for three-body potentials," Duke Math. J. 90, 379-434 (1997).

${ }^{14}$ Wada, T., "Scattering theory for time-dependent Hartree-Fock type equation," Osaka J. Math. 36, 905-918 (1999).

${ }^{15}$ Wang, X. P., "On the uniqueness of inverse scattering for $N$-body systems," Inverse Probl. 10, 765-784 (1994).

${ }^{16}$ Watanabe, M., "Inverse scattering for the nonlinear Schrödinger equation with cubic convolution nonlinearity," Tokyo J.

Math. 24, 59-67 (2001).

${ }^{17}$ Watanabe, M., "Uniqueness in the inverse scattering problem for the Hartree type equation," Proc. Jpn. Acad., Ser. A: Math. Sci. 77, 143-146 (2001).

${ }^{18}$ Watanabe, M., "Reconstruction of the Hartree-type nonlinearity," Inverse Probl. 18, 1477-1481 (2002).

${ }^{19}$ Weder, R., "Inverse scattering for the nonlinear Schrödinger equation," Commun. Partial Differ. Equ. 22, 2089-2103 (1997).

${ }^{20}$ Weder, R., " $L^{p}-L^{p^{\prime}}$ estimates for the Schrödinger equation on the line and inverse scattering for the nonlinear Schrödinger equation with a potential," J. Funct. Anal. 170, 37-68 (2000).

${ }^{21}$ Weder, R., "Inverse scattering on the line for the nonlinear Klein-Gordon equation with a potential," J. Math. Anal. Appl. 252, 102-123 (2000).

${ }^{22}$ Weder, R., "Inverse scattering for the nonlinear Schrödinger equation II. Reconstruction of the potential and the nonlinearity in the multidimensional case," Proc. Am. Math. Soc. 129, 3637-3645 (2001).

${ }^{23}$ Weder, R., "Inverse scattering for the nonlinear Schrödinger equation: Reconstruction of the potential and the nonlinearity," Math. Methods Appl. Sci. 24, 245-254 (2001).

${ }^{24}$ Weder, R., "Multidimensional inverse scattering for the nonlinear Klein-Gordon equation with a potential," J. Differ. Equations 184, 62-77 (2002).

${ }^{25}$ Weder, R., "Scattering for the forced non-linear Schrödinger equation with a potential on the half-line," Math. Methods Appl. Sci. 28, 1219-1236 (2005).

${ }^{26}$ Weder, R., "The forced non-linear Schrödinger equation with a potential on the half-line," Math. Methods Appl. Sci. 28 , 1237-1255 (2005). 